\title{
GESTAR Y PARIR EN PANDEMIA: VULNERACIÓN DE DERECHOS Y MARCAS SUBJETIVAS EN LA ATENCIÓN OBSTÉTRICA/PERINATAL EN CONTEXTO DE COVID-19 EN ARGENTINA
}

Pregnancy and Giving Birth during a Pandemic: Violation of Rights and Subjective Marks in Obstetric/Perinatal Care in the Context of Covid-19 in Argentina

Ana Inés Lázzaro ${ }^{1}$, Magdalena Arnao-Bergero ${ }^{2}$

Autora correspondencia: Ana Inés Lázzaro

Correo electrónico: anaineslazzaro@gmail.com

1. Doctora en Estudios Sociales en América Latina. Becaria Posdoctoral CONICET. Centro de Investigaciones y Estudios sobre Cultura y Sociedad (CIECS- CONICET). (Córdoba, Argentina)

ORCID: https://orcid.org/0000-0002-3967-4593

2. Doctora en Filosofía, especialista en Justicia de Género y Políticas Públicas. Docente investigadora de la Facultad Psicología Universidad Nacional de Córdoba y secretaria de Ciencia y Técnica de la Facultad de Filosofía y Humanidades (UNC). (Córdoba, Argentina)

ORCID: https://orcid.org/0000-0002-41265051

Recibido: 04/11/2020 Aceptado: 29/03/2021

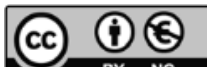




\section{RESUMEN}

OBJETIVO. Durante la emergencia sanitaria por COVID-19 la atención en torno a embarazos y partos se vio directamente afectada. Este estudio exploratorio indaga sobre las percepciones, emociones y vivencias de las personas gestantes en relación con la atención obstétrica en instituciones de salud de Argentina en los primeros meses del aislamiento social en este país. MATERIAL Y MÉTODO. Los datos fueron obtenidos mediante un cuestionario semiestructurado dirigido a personas gestantes y/o que hubieran parido desde el comienzo de la cuarentena. Para este artículo, nos basamos en el apartado cualitativo del mismo a partir del análisis de tres preguntas abiertas que apuntaban a registrar los efectos subjetivos de atravesar la gestación y/o parto durante la emergencia sanitaria. El enfoque teórico retoma los desarrollos en materia de derechos perinatales desde una perspectiva de género y estudios sociales sobre violencia obstétrica. RESULTADOS Y CONCLUSIONES. Se advierte la insistencia de emociones negativas y malestar por parte de la población encuestada, entendidos como «marcas subjetivas». Asimismo, se pone en relieve el estado de desinformación sobre protocolos de atención como factor clave para dicho malestar y el recrudecimiento en la vulneración de derechos perinatales (contemplados en marcos legislativos vigentes) como parte de los derechos sexuales y (no) reproductivos para todas las personas gestantes y con capacidad de gestar.

Palabras clave: Gestaciones y partos; Emergencia sanitaria; COVID-19; Subjetividades gestantes; Derechos perinatales; Violencia obstétrica.

\section{ABSTRACT}

GOAL. This study explores the perceptions, emotions and experiences of pregnant women in relation to obstetric care in health institutions in Argentina in the first months of social isolation in this country. MeTHODOLOGY. The data were collected by means of a semi-structured questionnaire directed to pregnant people and those who had given birth since the beginning of the quarantine. For this article, we focused on the qualitative section of the survey analyzing three open questions that aimed to register the subjective effects of going through pregnancy and delivery during the health emergency. Our theoretical approach takes up the developments in perinatal rights from a gender perspective and social studies on obstetric violence. RESULTS AND CONCLUSIONS. The results show the high frequency of stressful emotions and discomfort from the surveyed population, analyzed in terms of effects on the subjectivity. Likewise, it is very noticeable the state of misinformation about perinatal care protocols, as well as the worsening in the violation of perinatal rights (contemplated in current normative frameworks) as part of sexual and (non) reproductive rights for all pregnant people and with ability to gestate.

Keywords: Perinatal Rights; COVID-19; Sanitary Emergency; Pregnancies and Deliveries; Obstetric Violence; Pregnant Subjectivities. 


\section{INTRODUCCIÓN}

La emergencia social y sanitaria por el COVID-19 ha trastocado el orden de nuestros días dando paso a un estado de excepcionalidad en casi todas las esferas de nuestra vida social e íntima, afectando de manera material y subjetiva las mismas, a la vez que recrudeciendo múltiples situaciones de vulnerabilidad y desigualdad social (Vasconcelos et al., 2020). Frente a este escenario, son muchas las preguntas que se abren en torno a la convivencia de medidas sanitarias de emergencia y el respeto a los derechos de acceso a la salud.

Tal es el caso de la atención obstétrica/perinatal y los derechos de las personas gestantes en el marco de la reivindicación de los derechos sexuales y (no) reproductivos, cuestiones que tienen una larga trayectoria de denuncia social, dado los atropellos e imposiciones del «modelo médico hegemónico» sobre los cuerpos gestantes y con capacidad de gestar (Castro y Erviti, 2015; Sadler, et al., 2016; Borges, 2018).

En efecto, recordemos la extensa lucha proclamada a nivel mundial mediante el «movimiento transnacional por la humanización del embarazo, parto y nacimiento» (Tornquist, 2004) por el reconocimiento de los derechos durante el proceso gestacional y que, además, ha dado lugar a su enunciación en términos de «violencia obstétrica» como forma de violencia de género ejercida en la atención obstétrica/perinatal (Brigidi et al., 2019; Quattrocchi y Magnone, 2020).

Dichas reivindicaciones, en el caso de Argentina, se tradujeron en leyes específicas: Ley $n^{\circ} 29.929$ de «Derechos de Padres e Hijos en el Proceso de Nacimiento» y Ley $n^{\circ}$ 26.485 de «Protección Integral a las Mujeres» cuyo art. 6 refiere a la Violencia Obstétrica como violencia de género realizada por el personal de salud en la atención al embarazo, parto, postparto y postaborto. Aun cuando se trata de legislaciones vigentes, la emergencia sanitaria viene mostrando un panorama de vulneración de derechos perinatales como así también la ausencia de políticas públicas y datos oficiales concretos que permitan acceder a información clave para evaluar la situación y diseñar estrategias de abordaje e intervención efectivas.

En contraste, han ido creciendo los esfuerzos de organizaciones sociales, académicas, profesionales de la salud y activistas ligadas al campo de los derechos sexuales y (no) reproductivos, violencia obstétrica, parto respetado, maternidades y salud perinatal, etc. en pos de visibilizar algunos atropellos registrados en este marco y generar información 
e insumos para difundirlos, presentándose como claros indicadores de una situación compleja y confusa que demanda conocimiento.

La decisión del Estado argentino de realizar una cuarentena obligatoria comenzó el 20 de marzo de 2020. En esta situación de emergencia sanitaria, la atención en torno a embarazos y partos se vio directamente afectada. No obstante, se dieron algunos indicadores relevantes: la presentación de unas primeras «Recomendaciones para la atención de embarazadas y recién nacidos en contextos de pandemia» (en adelante Recomendaciones) que el Ministerio de Salud de la Nación lanza el 15 de abril del 2020 y que se vieron sucedidas por nuevas Recomendaciones el 20 de Julio de 2020 como así por las «Pautas para el cuidado de la Salud Perinatal y la Niñez en contexto de Pandemia» (en adelante Pautas) con fecha del 18 de mayo del 2020 (MSAL, 2020). Si bien las primeras Recomendaciones señalaban que no había - hasta ese momento- evidencia científica que justifique a las personas embarazadas y/o niño/as recién nacido/as como población de riesgo, las situaciones de desinformación, los reclamos de las gestantes y las advertencias en torno a la gravedad del contexto - en términos de derechos - por parte de colectivos y activistas nucleadas en torno a los derechos perinatales no dejaron de crecer, principalmente en relación a la falta de información, calidad de atención, acompañamiento y seguimiento médico de las gestantes y sus criaturas (Díaz Virzi, 2020; Centenar, 2020).

El presente trabajo surge, entonces, de la necesidad de generar datos sobre el estado de la cuestión en torno a gestaciones y partos que atraviesa la sociedad argentina en el contexto de emergencia sanitaria ante la pandemia por la COVID-19, tomando como eje el punto de vista y percepciones de las usuarias del servicio de atención obstétrico/perinatal. En tal sentido, y para este trabajo, retomamos las narraciones —en voz propia - de personas que atravesaron sus embarazos y/o partos durante los primeros meses de la cuarentena en Argentina, en pos de conocer y visibilizar algunas de las emociones y vivencias más acuciadas de las mismas y en relación con la dimensión de derechos.

Partimos de la hipótesis de que la emergencia sanitaria ha derivado en el recrudecimiento de prácticas y condiciones que conducen al ejercicio de la «violencia obstétrica» en la atención sanitaria, entendiendo que se trata de una problemática previa a la pandemia (Canevari, 2011; Jerez, 2015; Castrillo, 2019) pero cuya gravedad se ha profundizado, tal como sucede con otras violencias y desigualdades de género en este 
complejo escenario marcado por la COVID-19 (Vasconcelos et al., 2020). Asimismo, entendemos que esta forma de violencia afecta de manera directa y cotidiana la vida de miles de embarazadas teniendo consecuencias psicoemocionales que definimos en términos de «marcas subjetivas». Si bien este estudio no indaga sobre los efectos concretos de estas «marcas» en los embarazos y partos, diversos estudios confirman la centralidad de la emocionalidad para un desarrollo positivo los procesos gestacionales (Davenport, 2020; Arnao et. al, 2018; Borges, 2018; Buckley, 2015; Sadler, 2004).

Por último, y en tanto la «violencia obstétrica» — enmarcada en los derechos sexuales y (no) reproductivos - refiere a una violencia que no reconoce fronteras, tratándose, como refiere Oliden (2019), de una «epidemia global», inferimos que la información brindada por esta investigación es susceptible de potenciales cruces y comparación con otras regiones y para pensar futuros escenarios similares. En tal sentido, este trabajo busca transcender el conocimiento local ante un contexto específico para ser parte de un diagnóstico más vasto en materia de avances o estancamientos en materia de derechos sexuales y reproductivos y sus posibles efectos en las subjetividades gestantes.

\section{METODOLOGÍA}

\section{Instrumento de recolección y análisis de datos}

El presente artículo es el resultado del análisis de la fase cualitativa de un estudio más amplio. La recolección de datos se realizó mediante un cuestionario semiestructurado sobre el acceso a la salud obstétrica/perinatal en contexto de pandemia con miras a generar indicadores a partir de preguntas estandarizadas. En dicho cuestionario se incluyeron tres preguntas abiertas y a desarrollar que apuntaban a registrar las percepciones, emociones y vivencias de las gestantes y parturientas durante la cuarentena, dando lugar al relato de las usuarias del servicio de obstetricia. Las respuestas brindadas para tales interrogantes resultaron sumamente significativas en el momento de análisis, siendo retomadas como muestra de estudio para este trabajo.

Es importante aclarar que la toma de datos fue realizada de manera online (mediante la plataforma Google Forms). La selección de la muestra fue por bola de nieve, esto es, distribuido a personas que dieran con el perfil muestral a la vez que se difundió a través de redes sociales y otros soportes digitales. Partimos de la base de que este tipo de métodos de obtención de datos conllevan la necesidad de acceso a dispositivos 
tecnológicos y conectividad a Internet, lo cual implica un sesgo interseccional de clase. Algunos indicadores sociodemográficos reflejaron que, de un total de 370 participantes, la mayoría era adulta y poseía un nivel socioeconómico de clase media escolarizada, con predominancia en estudios superiores (ver Tabla 1).

TABLA 1. Características sociodemográficas de las participantes del estudio

\begin{tabular}{|c|c|c|}
\hline Variable & Modalidad & Valor \\
\hline \multicolumn{3}{|c|}{ Provincia de residencia } \\
\hline & $\begin{array}{l}\text { - Buenos Aires } \\
\text { - Córdoba } \\
\text {-Santa Fe } \\
\text { - Otras provincias }\end{array}$ & $\begin{array}{l}-136(36,8) \\
-110(29,7) \\
-98(26,5) \\
-26(7)\end{array}$ \\
\hline \multicolumn{3}{|l|}{ Edad } \\
\hline & $\begin{array}{l}-<20 \\
-20-30 \\
-30-40 \\
->40\end{array}$ & $\begin{array}{l}-15(4) \\
-138(37,3) \\
-195(52,7) \\
-22(5,9)\end{array}$ \\
\hline \multicolumn{3}{|c|}{ Nivel educativo alcanzado o en curso } \\
\hline & $\begin{array}{l}\text { - Posgrado: } \\
\text { - Universitario: } \\
\text { - Terciario: } \\
\text { - Nivel medio: } \\
\text { - Nivel primario: }\end{array}$ & $\begin{array}{l}-52(14) \\
-153(41,3) \\
-106(28,6) \\
-58(15,7) \\
-1(0,3)\end{array}$ \\
\hline \multicolumn{3}{|c|}{ Trabajo remunerado } \\
\hline & $\begin{array}{l}\text { - Sí: } \\
\text { - No: } \\
\text { - A veces: }\end{array}$ & $\begin{array}{l}-225(60,8) \\
-109(29,5) \\
-36(9,7)\end{array}$ \\
\hline \multicolumn{3}{|c|}{ Seguro social o cobertura de salud prepaga } \\
\hline & $\begin{array}{l}\text { - Sí: } \\
\text { - No: }\end{array}$ & $\begin{array}{l}-297(80,3) \\
-73(19,7)\end{array}$ \\
\hline \multicolumn{3}{|c|}{ Dónde realizaba controles obstétricos } \\
\hline & $\begin{array}{l}\text { - Ámbito privado: } \\
\text { - Ámbito público: }\end{array}$ & $\begin{array}{l}-279(75,3) \\
-91(24,6)\end{array}$ \\
\hline \multicolumn{3}{|c|}{ Situación de convivencia durante la cuarentena } \\
\hline & $\begin{array}{l}\text { - En convivencia } \\
\text { - Solas }\end{array}$ & $\begin{array}{l}-328(88,6) \\
-42(11,4)\end{array}$ \\
\hline
\end{tabular}

El periodo de circulación del cuestionario fue desde el 27 de abril de 2020 hasta el 27 de julio de 2020 (durante la primera etapa de la cuarentena), cuyo cierre se dio de manera arbitraria con el fin de generar un primer corte de análisis y estado general de la cuestión. 
Las respuestas obtenidas para esta primera etapa fueron respondidas por habitantes de las grandes urbes: Buenos Aires, Córdoba y Santa Fe, lo cual supone una limitación respecto alcance nacional de las respuestas como también acerca del estado de la cuestión en zonas rurales del país.

La encuesta fue anónima y todas las participantes dieron su consentimiento mediante un apartado previo al cuestionario en el cual se explicitaron los objetivos y responsables de la investigación así como el uso de las respuestas proporcionadas con fines científicos. ${ }^{1}$

Las preguntas abiertas y a desarrollar sobre las cuales versa este escrito fueron las siguientes:

1. Comenta cómo estás viviendo o vivenciaste tu embarazo desde el comienzo de la pandemia (emociones, sensaciones, experiencias).

2. Comenta lo que consideres más significativo de la experiencia de tu parto.

3. Describe brevemente cómo estás atravesando el puerperio en contexto de pandemia.

El análisis de datos se realizó mediante el análisis de contenido (Bardin, 1996), sistematizando recurrencias y redundancias en las respuestas para la construcción de indicadores y categorías en tanto líneas de interpretación. A partir de esta técnica se consolidaron tres instancias: a) Observación preliminar, en donde se organizó y ordenó el material y a partir de lo cual surgieron las primeras líneas de análisis; b) Exploración exhaustiva del material, analizando las repuestas de manera individual y agrupándolas función de las redundancias/regularidades encontradas. Subtareas: elección de unidades de análisis (conteo, clasificación y agregación), elección de categorías descriptivas; c) Por último se realizó el tratamiento de datos e interpretaciones pertinentes, identificando nuevos interrogantes a seguir trabajando en este escenario tan complejo como cambiante.

\footnotetext{
${ }^{1}$ En la primera parte del cuestionario, y para continuar a las respuestas, se pedía autorización a las participantes mediante el siguiente párrafo: «Su participación en el estudio es voluntaria, anónima y no le expone a ningún riesgo. Los datos serán mantenidos en confidencialidad y serán resguardados bajo secreto estadístico. Al completar este cuestionario entiendes que estos datos se utilizarán con fines científicos, aceptas el procesamiento de respuestas de acuerdo con la información aquí proporcionada y cuya publicación anónima busca promover la transparencia en la ciencia y el diseño de políticas públicas». Por su parte, el equipo «Mi cuerpo mi decisión» perteneciente a la Facultad de Psicología de la Universidad Nacional de Córdoba (Argentina) fue el encargado de revisar los aspectos éticos y metodológicos del estudio.
} 


\section{RESULTADOS Y DISCUSIÓN}

Este análisis retoma el apartado cualitativo del cuestionario focalizándose en los relatos proporcionados en las preguntas abiertas y a desarrollar. Cabe aclarar que, dichas preguntas no contaban con límites de extensión por lo que las participantes podían expresarse de manera extendida. Las citas en cursiva y entrecomilladas son tomadas textualmente de las respuestas, consignándose, además, la edad, si tenían o no cobertura de salud (CC, SC respectivamente) y si eran atendidas en una entidad pública o privada (PU y PRI respectivamente). Ejemplo: 38, SC, PU (38 años, sin cobertura, se atiende en entidad pública). El género autopercibido mayoritariamente fue femenino, razón por la cual este dato quedará sobreentendido para todas las respuestas. En función del conteo, clasificación y agregación del análisis de contenido se consolidaron 4 grupos temáticos predominantes:

\section{«Emociones encontradas»}

En este subtema se destacan las principales emociones y experiencias que relatan las informantes en relación con el embarazo durante la pandemia ante la pregunta «Comenta cómo estás viviendo o vivenciaste tu embarazo desde el comienzo de la pandemia (emociones, sensaciones, experiencias)». Aun cuando en algunos relatos se hace referencia al vaivén emocional con expresiones del tipo «con altibajos», «un tren de emociones», «muchas emociones encontradas», las emociones más acuciadas son: miedo, incertidumbre, ansiedad, preocupación y sensación de abandono. Si bien podría decirse que muchas de estas emociones son típicas en y durante los procesos gestacionales (Lázzaro, 2020), llama la atención la predominancia que adquieren en la gran mayoría de los testimonios, mientras que otras emociones son escasamente referidas. En función del análisis cualitativo traducimos dichos resultados a un mapa semántico (Gráfico 1) que visibiliza algunos de los datos sobresalientes en términos de marcas subjetivas. 
Gráfico1. Nube de palabras de las emociones más frecuentes registradas en los relatos

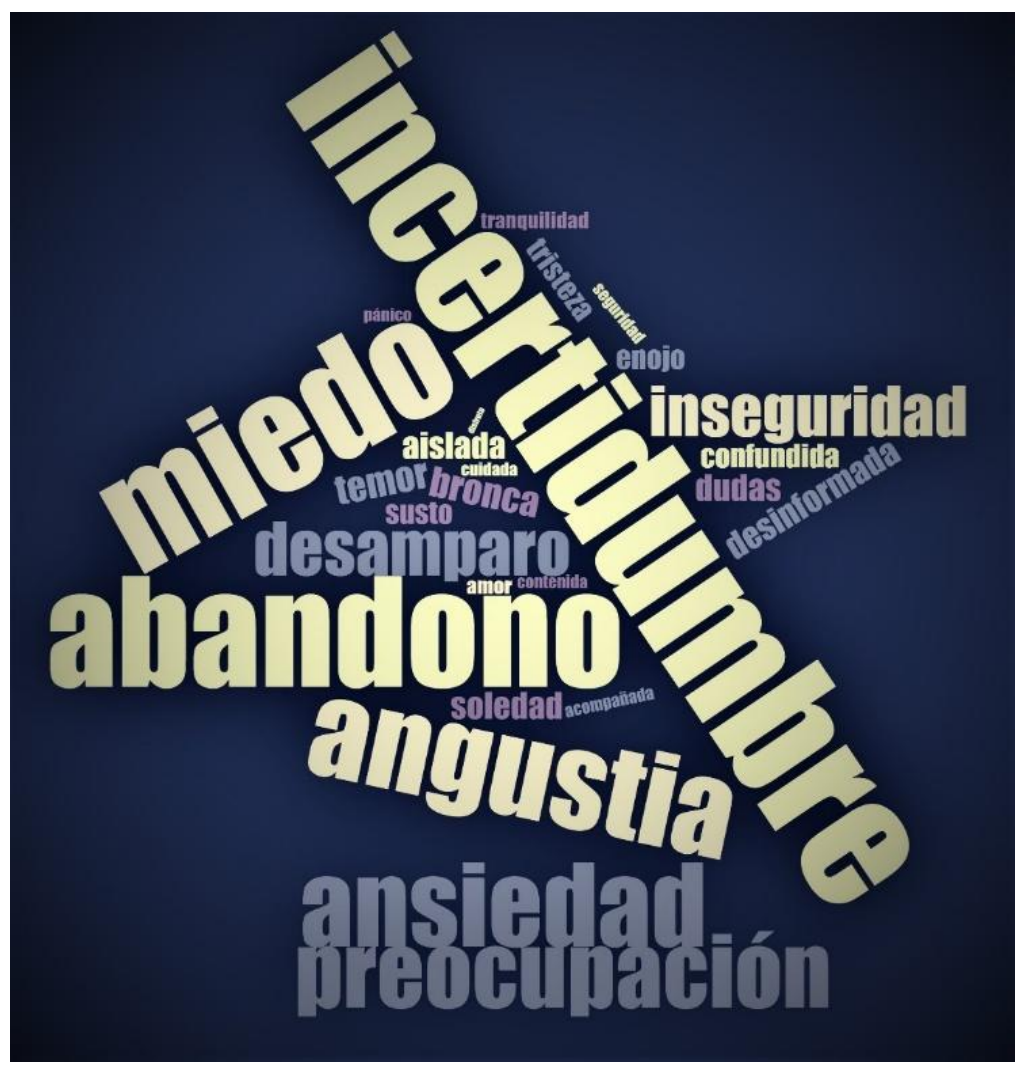

Conviene destacar que estas emociones - entendidas como negativas o estresoras - no solo refieren a vivir un embarazo, parto y/o posparto en pandemia (por ejemplo, cuestiones estrictamente sanitarias y/o de contagio), sino en relación con los aspectos económicos, laborales y también vinculares-afectivos, puesto que inciden directamente en estados emocionales y, por ende, en la gestación como tal (Buckley, 2015; Olza Fernández, 2019).

Acompañan estos datos los relatos de las participantes, donde podemos advertir los significantes de algunos de los términos relevados anteriormente:

Un tren de emociones, preocupación por el parto, miedo al contagio en un lugar que debiese ser seguro para mí y mi bebe que nacerá, no me están realizando los controles por la entidad pública correspondiente, eso causa una mayor inseguridad al no saber cómo va el crecimiento del bebe ya que no estoy con trabajo, a mi pareja la despidieron de su actual trabajo por ende no podemos pagar un médico de forma particular. (27, SC, PU)

Aumenta la sensación de angustia y preocupación frente a respuestas poco claras por parte de las instituciones médicas, librando a la total responsabilidad a las decisiones de los padres en un contexto incierto y extraño. (38, CC, PRI) 
En cuanto a la pregunta de «Cómo están atravesando su puerperio en contexto de pandemia» (destinada específicamente a quienes ya hubieran parido durante la cuarentena), vuelven a aparecen expresiones que remiten al miedo (especialmente de contagio), pero las emociones más acuciadas en estos relatos son angustia y soledad. En este sentido, las referencias a la incertidumbre se atenúan en comparación al embarazo, acentuándose aquí la sensación de malestar que genera el encierro y aislamiento social, especialmente en relación con los vínculos afectivos cercanos:

Mucho miedo... es mucha ansiedad encerrada. (32, CC, PRI)

El puerperio es una montaña rusa de emociones y vivirlo en una situación de encierro involuntario es una tarea difícil. (29, CC, PRI)

De todas maneras, en algunos testimonios se hace referencia al hecho de que, a raíz de la cuarentena, pudieron vivir esta etapa en compañía con el apoyo y colaboración de sus familiares, quienes de otra manera estarían fuera del hogar. Lo dicho pone en relieve la necesidad de un debate — que trasciende este contexto- acerca de las licencias de ma(pa)ternidad como de la importancia de atravesar un puerperio con ayuda material, económica y sostén afectivo.

\section{Cambios en la atención}

La emergencia sanitaria supuso reestructuraciones inmediatas en la atención hospitalaria, su funcionamiento y dinámicas. Esta situación repercutió enormemente en la atención obstétrica/perinatal, la cual está centralizada en dispensarios, hospitales y clínicas. En este escenario, muchas gestantes quedaron tan «desatendidas» como «desinformadas» sobre los nuevos protocolos de atención (en los casos de haberlo), refiriendo en varias ocasiones a una situación de «abandono» por parte de la institución:

Me suspendieron todo, hasta casi ni información se, ni tengo para expresar porque no me decían nada y no sé nada! (20, SC, PU)

Incertidumbre y me sentí totalmente abandonada por el sistema de salud. (35, CC, PRI)

Desprotegida. Ya que al atenderme en hospital público ni médicos ni pediatras atienden si no es por una emergencia. (28, SC, PU)

Con mucha incertidumbre y angustia de no saber cómo proceder, falta de acompañamiento profesional, mucha carga emocional. En mi caso la obra 
social dejo de darme la posibilidad de cobertura dejándome afuera de los planes maternales. $(25, \mathrm{CC}, \mathrm{PRI})$

Sin lugar a duda, esta dimensión informativa se vincula estrechamente a la emocional, ya que supone la discontinuidad en el seguimiento del embarazo, a la vez que implica la necesidad de resolver cuestiones de tipo operativo (conseguir turnos para control o exámenes, autorizar órdenes, etc.) en pleno aislamiento social. Se ponen, así, en relieve los impactos negativos del proceder de las instituciones ante las reestructuraciones de la atención durante la emergencia sanitaria incluso en los casos de embarazos de riesgo:

Tenía presión alta y diabetes gestacional me habían programado cesárea para el primero de abril y cuando surgió la cuarentena dejaron de atenderme. (28, $\mathrm{SC}, \mathrm{PU})$.

Mi embarazo fue triple hasta la semana 7. Supuestamente iba a tener mayores controles y seguimiento por ser de riesgo, pero solo tuve ecografía en la semana 16 y voy a tener controles y consulta recién en la semana 23. (37, CC, PRI).

Me preocupó mucho que me suspendieran los controles justo en las semanas claves antes del parto (37 en adelante). Nunca me avisaron que había un procedimiento especial para personas en mi situación de embarazo de riesgo, luego lo encontré en la página de la clínica con comunicado general, nunca me contactaron personalmente. (31, CC, PRI)

Tengo un embarazo gemelar y me da bastante miedo esto de no recibir atención médica, los controles. Todo lo hago por mi cuenta. No puedo ir al hospital porque vivo sola con mis hijos. El centro de salud más cercano cerró. El ginecólogo más cercano que tengo no se reincorporo de su licencia debido al aislamiento. No sé qué hacer. Intento conservar la calma y confiar en mi cuerpo como lo hice antes, es mi único recurso. (39, SC, PU)

Además de evidenciar el efecto estresor sobre las subjetividades que supone la falta de atención (y de información), estas cuestiones plantean el interrogante de sobre quiénes recae - en última instancia - la responsabilidad del estado de salud de la embarazada y su criatura: «librando la total responsabilidad a las decisiones de los padres en un contexto incierto y extraño», como decía uno de los testimonios más arriba. Aun cuando, históricamente, el modelo médico de atención perinatal ha impedido la participación activa y protagonismo de las mujeres en sus procesos gestacionales, precisamente, por considerar el riesgo como factor que define - a priori y para todos los casos - los embarazos y partos; razón por la cual se justifica el marcado intervencionismo y medicalización de los mismos (Arnau Sánchez et al, 2017; Montero y Leida, 2017). 


\section{Gestaciones, nacimientos y COVID-19}

Además de la continuidad de los protocolos de atención, otro de los aspectos que contribuyen a la dimensión informativa tiene que ver con las relaciones y efectos del COVID-19 en el embarazo, parto, posparto. La mayoría de los relatos refieren a una situación de desconocimiento e incertidumbre:

Mucha incertidumbre! Momentos de angustia y desconcierto sin saber qué es lo mejor de hacer. (37, CC, PRI)

Mucha incertidumbre, consultas rápidas y respuestas insuficientes. NO se nombra NADA sobre el acompañamiento en parto/cesárea. Análisis que se siguen postergando. Ya no confío en el lugar que elegí para parir. Todo repercute a nivel emocional generando angustia y miedo a que no se cumplan mis derechos. (31, SC, PU)

Solas, aisladas y desinformadas. (27, SC, PU)

Horrible, mucha angustia. Quería un parto respetado pero ya me hice la idea de entregarme a la situación y que sea lo mejor. (41, CC, PRI)

Esta situación también es referida por las informantes que estaban atravesando un puerperio, aunque en menor medida, pues la atención pediátrica a domicilio - para quienes son usuarias del servicio de salud privado- aparece como un atenuante de la incertidumbre sobre el estado de salud de la criatura. No obstante, para quienes hacen dichos controles en instituciones públicas, se repiten sensaciones de malestar debido a las suspensiones de los controles neonatológicos y desconocimiento de los protocolos, especialmente en relación con los traslados y/o al contagio:

Salir para los controles del bebé es muy estresante y angustiante. No hay permisos para este tipo de salidas y la policía no siempre entiende la situación. Viví varios momentos angustiantes con policías que cuestionaron la salida por controles neonatales indicados por médicos. A eso se suma la angustia por el riesgo a enfermarme. (31, CC, PRI)

En definitiva, el hecho de que los índices de incertidumbre, ansiedad, miedo $-\mathrm{y}$ emociones asociadas - sean elevados responde a una superposición de planos/capas que sin duda se influyen recíprocamente: se trata de emociones típicamente vinculadas a los procesos gestacionales, sumadas a las condiciones y reestructuraciones de los hospitales y clínicas dada la emergencia sanitaria y que impactaron en la continuidad atención sin ser, además, debidamente informadas a las usuarias. Asimismo, cabe añadir la escasa información que reciben en relación con embarazo/puerperio en el contexto de la COVID- 
19 (implicaciones, riesgos, procedimientos), las consecuencias laborales y económicas de la cuarentena que, como han revelado algunos estudios, impactan especialmente en el colectivo de mujeres (ONU, 2020), el encierro e imposibilidad de cercanía con familiares y afectos, en un un proceso donde el sostén vincular-emocional es clave, entre otras.

\section{Parir solas}

En muchos testimonios de quienes aún estaban cursando el embarazo, se hace referencia a la preocupación, angustia y temor de tener que "parir solas», esto es, sin un acompañante de su elección, un derecho básico contemplado en la ley 29.929 de Parto Humanizado/Respetado de este país en consonancia con posicionamientos internacionales (OMS, 2014). De acuerdo con las Recomendaciones y Pautas, no habría motivo para impedir dicho acompañamiento, no obstante, el contexto sanitario fue motivo para que las instituciones de salud vulneraran, de manera inapelable, este derecho (Bagliardelli, 2020; Ríos, 2020; Simmone, 2020).

De aquí la sensación de malestar que se advierte ante la posibilidad de tener que parir sin una compañía afectiva en los relatos de quienes aún estaban gestando durante la emergencia sanitaria:

La angustia e incertidumbre de que mi pareja no pueda acompañarme en los controles y no saber si va a estar en el parto es muy angustioso. (29, CC, PRI).

Con preocupación respecto al protocolo ya que no han informado si a la hora de parir puedo contar o no con mi acompañante. La información es escasa y todo el tiempo va cambiando lo que lleva a más ansiedad. (31, CC, PRI)

En general es muy difícil y solitario. Mucha preocupación por posibles dificultades en el embarazo y bienestar de la bebé (distintos a Coronavirus). En la Clínica del Sol las ecografías fueron sola. En el momento del parto o cesárea, si sigue la cuarentena, voy a estar completamente sola, esto me desespera, no creo poder. La opción que tenemos es abonar una habitación privada ( $\$ 5000$ por día). Este embarazo, a diferencia del anterior, lo considero muy complicado y he tenido momentos de mucha tristeza, desesperanza y sin ganas de seguir. (37, CC, PRI)

Con muchas dudas. Me dicen que mi marido no podrá entrar. Y fue ahí donde me puse a leer sobre la ley. Estoy muy angustiada ya que me quedan dos semanas a la fecha posible de parto y no creo que estén cumpliendo con la ley al no dejar entrar a mí marido conmigo. Esto me tiene muy angustiada. (33, $\mathrm{SC}, \mathrm{PU})$ 
Y también para quienes parieron en durante la emergencia sanitaria:

A la hora de tener el bebé, fue un parto totalmente no respetado, solitario, 3 días sola sin compañía, trabajo de parto sin si quiera un enfermero, no me dieron líquidos, no me acompañaron en nada del proceso, me abandonaron, hoy depresión post parto por el trauma del parto. Me trato con psicóloga y psiquiatra perinatal para poder superar todo eso. (31, CC, PRI)

Fue terrible todo lo vivido, solo ver a mi hijo fue lo único emotivo en tres días de soledad de la internación. (23, CC, PU)

Lo dicho destaca nuevamente la importancia que asume la dimensión afectivovincular para atravesar estos procesos de manera positiva, cuestión que forma parte de las recomendaciones de la OMS como también de las legislaciones mencionadas; mientras que su desconsideración es asumida como uno de los indicadores básicos de violencia obstétrica. (Sadler, 2004)

\section{CONCLUSIONES}

En estas páginas, se presentaron algunas de las marcas subjetivas —expresadas en emociones, percepciones y experiencias - sobre el embarazo, parto y la atención obstétrica/perinatal desde el inicio de la emergencia sanitaria por la COVID-19 en Argentina. Una cuestión que emerge de los datos relevados es la estrecha relación entre la información y la dimensión emocional de las gestantes. Se pone así en evidencia las consecuencias negativas (malestar, angustia, temores, etc.) de la falta de información en distintos niveles. Esta tendencia también coincide con las denuncias realizadas por las organizaciones y activistas de los derechos perinatales.

De aquí resulta que muchas de las sensaciones acuciadas sean «abandono», «desamparo» por parte de las instituciones, acarreando preocupaciones, temores, sensación de soledad y poca contención, aun cuando la gran mayoría de las encuestadas refirió a estar acompañada por alguien de su entorno íntimo durante la cuarentena. Este impacto no es casual, ya que, históricamente, el modelo médico de atención perinatal se caracterizó por una impronta intervencionista y de tutelaje paternalista sobre las gestantes, dejándolas en un lugar subsidiario para tomar decisiones y participar activamente sobre sus procesos. En este sentido, resulta alarmante cómo un modelo de atención caracterizado por el monitoreo y control continuo sobre las gestaciones y partos dejó a 
las pacientes sin atención médica ni información al respecto en un momento tan particular e incierto como fueron las medidas de aislamiento social durante la emergencia sanitaria.

¿Qué hizo posible que el sistema de salud responda con desinformación y desamparo a las usuarias? Más aun, en el escenario que se configuró durante la pandemia en el 2020 y de cara a la continuidad de la misma y a la llamada «nueva normalidad». ¿Podemos pensar en escenarios de alta complejidad sanitaria que no sean terreno de justificación del retroceso y vulneración de derechos? ¿Qué alcance tienen las marcas subjetivas producto del abandono y atropellos que aquí se registran? Esta situación demanda la necesidad de visibilizar y sensibilizar a la población sobre la violencia obstétrica y sus impactos sobre las subjetividades gestantes como también la urgencia de políticas públicas para la consolidación de mecanismos y estrategias que garanticen los derechos perinatales aún en situaciones de emergencia, de modo integral y desde una perspectiva de género.

\section{AGRADECIMIENTOS}

La elaboración de este estudio así como sus criterios éticos fueron supervisados por los miembros del equipo de investigación «Mi Cuerpo, Mi decisión» perteneciente a la Facultad de Psicología Universidad Nacional de Córdoba (Argentina). 


\section{BIBLIOGRAFÍA}

Arnao Bergero, M.; Galván, V. L.; Rosso, F. Parir y nacer. Trazas corporales, impacto subjetivo y derechos vulnerados. En: Revista de Psicología. 2018, vol. 17, núm. 2, pp. 3-13.

ARNAU SÁnCHEZ, J., et al. Análisis etnográfico del intervencionismo en la atención perinatal. En: Index de Enfermería. 2017, vol. 26, núm. 4, pp. 270-274.

Bagliardelli, M. Dar a luz en tiempo de pandemia ¿Con más o menos derechos? En: Qué portal de contenidos: 18 de mayo 2020. [Consulta 30 de octubre 2020]. Disponible en: https://bit.ly/3iKOHvJ

BARDIN, L. Análisis de contenido. Madrid: Akal, 1996.

Borges, M. T. A Violent Birth: Reframing Coerced Procedures During Childbirth as Obstetric Violence. En: Duke Law Journal. 2018, vol. 67, núm. 4, pp. 827-862.

Brigidi, S., et al. Mirades a la Violència Obstètrica. Barcelona: Pol·len Edicions, 2019.

BUCKLEY, S. J. Executive summary of hormonal physiology of childbearing. Evidence and implications for women, babies and maternity care. En: The journal of perinatal education. 2015, vol. 24, núm. 3, pp. 145-153.

CANEVARI, C. Cuerpos enajenados: experiencias de mujeres en una maternidad pública. Santiago del Estero: Barco Edita, 2011.

CASTRILlo, B. Hacer partos y parir: hacia una sociología de la atención médica en embarazos $y$ partos. Experiencias de mujeres-madres, varones-padres $y$ profesionales de la salud en La Plata (2013-2019). [Tesis doctoral]. Facultad de Humanidades y Ciencias de la Educación, Universidad Nacional de La Plata, Argentina: 2019. [Consulta: 10 de mayo 2020]. Disponible en:

http://sedici.unlp.edu.ar/handle/10915/78629

Centenar, M. Partos en casa, una opción contra el miedo al coronavirus en Argentina. [En línea]. En: El país: 10 de mayo 2020. [Consulta: 15 de mayo 2020]. Disponible en: https://bit.ly/3gyrP14

CASTRO, R.; ERVITI, J. Sociología de la práctica médica autoritaria. Violencia obstétrica anticoncepción inducida y derechos reproductivos. México: CRiM UNAM, 2015.

Davenport M. H, et al. Moms Are Not OK: COVID-19 and Maternal Mental Health. En: Frontiers in Global. Womens Health. 2020, vol. 1, num. 1.

DIAZ VIRZI, S. Abrazos pospuestos, amor «virtual» e incertidumbre: gestar (y parir) durante la pandemia. [En línea]. En: Diario Clarín. 1 de julio 2020. [Consulta: 9 de julio 2020]. Disponible en: https://bit.ly/3gugJKc 
JEREZ, C. Partos «humanizados», clase y género en la crítica a la violencia hacia las mujeres en los partos. [Tesis de licenciatura]. Facultad de Filosofía y Letras, Universidad Nacional de Buenos Aires, Argentina: 2015. [Consulta: 12 de abril 2020]. Disponible en:

http://antropologia.filo.uba.ar/sites/antropologia.filo.uba.ar/files/documentos/Jerez \%20-\%20Tesis.pdf

LÁzzaro, A. I. Dispositivos Instituyente: entre la heteronomía instituida y la construcción de autonomía. Una aproximación a rondas que promueven el parto respetado en Córdoba. Córdoba: Editora CEA-UNC, 2020. [Consulta: 10 de julio 2020]. Disponible en: https://rdu.unc.edu.ar/handle/11086/15927?show=full.

Montero, C.; LeIDA, C. El parto y el nacimiento en la modernidad. Una visión con perspectiva de género desde la enfermería obstétrica. En: Comunidad y Salud. 2017; vol. 15 , num. 1 , pp. $42-52$.

Ministerio de SAlud de la Nación Argentina (MSAL). Nuevo Coronavirus COVID 19. Información, recomendaciones del Ministerio de Salud de la Nación y medidas de prevención. [En línea]. MSAL: 2020. [Consulta: 2 de noviembre 2020]. Disponible en: https://bit.ly/35zNn6R

OLIDEN, A. Violencia obstétrica: una epidemia global. En: Mirades a la violència obstètrica. Barcelona: Pol.len edicions, 2019, pp. 9-34.

OLZA-FERNÁNDEZ, I. El trastorno de estrés postraumático como secuela obstétrica. En: Cuadernos De Medicina Psicosomática y Psiquiatría De Enlace. 2019; vol. 23, núm. 96, pp. 35-41.

Organización Mundial de la SAlud (OMS). Prevención y erradicación de la falta de respeto y el maltrato durante la atención al parto en centros de salud. [En Línea]. Ginebra: OMS, 2014 [Consulta: 26 de Julio 2020]. Disponible en: https://bit.ly/3cOzgid

Organización Mundial de la SAlud (OMS). Manejo clínico de COVID-19. Orientación Provisional. [En línea]. OMS: 27 mayo 2020. [Consulta: 8 de agosto 2020]. Disponible en: https://www.who.int/publications/i/item/clinical-managementof-covid-19

ORganización de las NACIONES UnidAs (ONU). COVID-19 en América Latina y el Caribe: cómo incorporar a las mujeres y la igualdad de género en la gestión de la respuesta a la crisis. [En línea]. ONU: 2020. [Consulta: 8 de agosto 2020]. Disponible en: https://ac.unwomen.org/es/digiteca/publicaciones/2020/03/covidcomo-incorporar-a-las-mujeres-y-la-igualdad-de-genero-en-la-gestion-de-respuesta

ObSERVATORio Violencia ObStÉtrica Argentina (OVO). Informe final. OVO: 2015. [Consulta: 26 de Julio 2020]. Disponible en: https://drive.google.com/file/d/0BucL20WyuYRYTRZWThPcWVyY1U/view. 
Organización Panamericana de la SAlud (PAHO). Salud materno-perinatal y Covid-19. [En línea]. Abril 2020. [Consulta: 8 de agosto 2020]. Disponible en: https://www.paho.org/clap/images/PDF/presentacionparteras/Presentacin_CLAP_C OVID19 abril_2020.pdf?ua=1

Quattrocchi, P.; Magnone, N. (eds). Violencia Obstétrica en América Latina: conceptualización, experiencias, medición y estrategias. Buenos Aires: Colección Cuadernos del ISCo, UnLa; 2020.

Ríos, L. La violencia obstétrica y derechos en el parto durante la pandemia. [En línea]. En: Agencia Telam: 17 de abril 2020. [Consulta: 3 de mayo 2020]. Disponible en: https://www.telam.com.ar/notas/202004/452745-violencia-obstetrica-partospandemia.html

SADler, M. Así me nacieron a mi hija. Aportes antropológicos para el análisis de la atención biomédica del parto. En: Sadler, Acuña; Obach (comp.) Nacer, Educar, Sanar; Miradas desde la Antropología del Género. Santiago de Chile: Colección Género, Cultura y Sociedad, 2004, pp. 15-66.

SADLER, M., et al. Moving beyond disrespect and abuse: addressing the structural dimensions of obstetric violence. En: Reproductive Health Matters. 2016, vol. 24, núm. 47, pp. 47-55.

Simmone, S. Gestar y parir en Entre Ríos en tiempos de coronavirus. En: Mirador Entre Ríos. 10 de mayo 2020. [Consulta: 13 de mayo 2020] Disponible en: https://www.miradorprovincial.com/?m=interior\&id um=238914-gestar-y 10-pariren-entre-rios-en-tiempos-de-coronavirus-embarazo-y-maternidad

Vasconcelos M., et al. (orgs.) Janelas da Pandemia. Belo Horizonte: Editora Instituto DH, 2020. 